\title{
Recurrence plot analysis of DNA sequences
}

\author{
Zuo-Bing $\mathrm{Wu}$ \\ State Key Laboratory of Nonlinear Mechanics, Institute of Mechanics, Chinese Academy of Sciences, Beijing 100080, China \\ Received 3 June 2004; received in revised form 9 September 2004; accepted 14 September 2004 \\ Available online 7 October 2004 \\ Communicated by C.R. Doering
}

\begin{abstract}
Recurrence plot technique of DNA sequences is established on metric representation and employed to analyze correlation structure of nucleotide strings. It is found that, in the transference of nucleotide strings, a human DNA fragment has a major correlation distance, but a yeast chromosome's correlation distance has a constant increasing.
\end{abstract}

(C) 2004 Elsevier B.V. All rights reserved.

Keywords: DNA sequences; Recurrence plot; Correlation structure

\section{Introduction}

The heredity and variation information of all organisms is embodied in DNA sequences. To understand the one-dimensional symbolic sequence made of four letters $A, C, G$, and $T$, some statistical and geometrical methods are developed in bioinformatics. Chaos game representation (CGR) [1], which generates a two-dimensional square from a one-dimensional sequence, provides a technique to visualize the composition of DNA sequences. The characteristics of CGR image is described as genomic signature [2]. A visualization scheme of the string composition of DNA sequences is proposed and used to trace evolutionary relatedness of species $[3,4]$. Recently, a one-to-one

E-mail address: wuzb@1nm.imech.ac.cn (Z.-B. Wu). metric representation (MR) $[5,6]$ is proposed to make an ordering of subsequences in a plane and determine suppression of certain nucleotide strings in DNA sequences. By using the MR method, self-similarity limits of genomic signatures are determined as optimal string lengths for generating the genomic signatures [6].

For a chaotic system, the dynamical trajectory is always attracted in a finite set. To depict the finite set, i.e., measure its self-similarity, information dimension is designed [7]. At the same time, to describe the ergodicity of trajectory, i.e., reflect natural time correlation information of dynamical system, a recurrence plot technique is presented [8]. The methods can be used to diagnose unknown dynamical information from an experimental time series. Especially, by using the tool of recurrent plot, dynamical assessment of physiological systems is illustrated [9], nonrandom 
dynamical components in synaptic noise of central neurons are evidenced [10] and structure-function relationships of proteins are quantified [11].

In DNA sequences, correlation structure of nucleotide strings, as an important part of the DNA architecture, is covered in the transference of nucleotide strings. In addition, DNA transposable elements, which are found in all organisms, have ability to move from place to place and make many copies within the genome via the transposition $[12,13]$. In general, the correlation structure of nucleotide strings can provide an understanding of the elements transposition in an extensive region. In this Letter, using the MR method, we extend recurrence plot technique to analyze correlation structure of nucleotide strings and determine transference of nucleotide strings.

\section{Method}

A DNA sequence is a one-dimensional symbolic sequence $s_{1} s_{2} \ldots s_{i} \ldots s_{N}\left(s_{i} \in\{A, C, G, T\}\right)$. In a two-dimensional MR, we make a correspondence of points $(\alpha, \beta)$ and subsequences $\Sigma_{m}=s_{1} s_{2} \ldots s_{m}$ $(1 \leqslant m \leqslant N)$. Subsequences with the same ending $k$-nucleotide string, which are labeled by $\Re_{k}$, correspond to points $(\alpha, \beta)$ in the zone encoded by the $k$-nucleotide string. For a given $k$-nucleotide string, we have a set $\Re_{k}$ and a correspondent zone size $\epsilon_{k}=3^{-k}$. Taking a subsequence $\Sigma_{i} \in \Re_{k}$, we calculate

$$
\begin{aligned}
& \Theta\left(\epsilon_{k}-\left|\Sigma_{i}-\Sigma_{j}\right|\right) \\
& \quad=\Theta\left(\epsilon_{k}-\sqrt{\left(\alpha_{i}-\alpha_{j}\right)^{2}+\left(\beta_{i}-\beta_{j}\right)^{2}}\right),
\end{aligned}
$$

where $\Theta$ is the Heaviside function $[\Theta(x)=1$, if $x>0 ; \Theta(x)=0$, if $x \leqslant 0$ ] and $\Sigma_{j}$ is a subsequence $(k \leqslant j)$. When $\Theta\left(\epsilon_{k}-\left|\Sigma_{i}-\Sigma_{j}\right|\right)=1$, i.e., $\Sigma_{j} \in \Re_{k}$, we plot a point $(i, j)$ in a plane. If $\Sigma_{j}$ is taken from the beginning of one-dimensional symbolic sequence and shifted forward, we plot all correspondent points in the plane and obtain some points at the position $i$. When the position $i$ is moved from the beginning of one-dimensional symbolic sequence, $\Sigma_{i}$ belongs to another $k$-nucleotide string set. Repeating the above process, we obtain a recurrence plot of the DNA sequence. In the recurrent plot, there exists a mirror symmetry with respect to the diagonal $i=j$. A point in the recurrence plot means that two subsequences $\Sigma_{i}$,
$\Sigma_{j} \in \Re_{k}$ have a distance $|j-i|$ in the DNA sequence. Along with the increase of $k$, each zone size in the MR plane decreases. For a given $\Sigma_{i}$, neighboring points (encoded by $\Sigma_{j}$ ) of its correspondent point in the zone decrease, so that the points $(i, j)$ in the recurrence plot plane decrease.

To quantify the correlation structure in recurrence plot plane, we define a correlation intensity at a given correlation distance $l$

$$
\Xi(l)=\sum_{i=1}^{N-l} \Theta\left(\epsilon_{k}-\left|\Sigma_{i}-\Sigma_{i+l}\right|\right) .
$$

The quantity displays the transference of all $k$-nucleotide strings with the correlation distance $l$ in the DNA sequence.

\section{Results}

\subsection{Correlation structure of $H U M H B B$}

To display properties of recurrence plots, we take HUMHBB (human $\beta$-region, chromosome 11) with 73308 bases as an example and draw the recurrence plots for $k=7,9,11,13$ and 15. Along with the increase of $k$, a point density in the recurrence plot plane decreases monotonically. The recurrence plot with a high density is easier to investigate global properties than that with a low density, but to find local properties such as the transference of long nucleotide strings, latter is better. In Fig. 1(a), a recurrence plot of HUMHBB for $k=9$ is plotted. Beside a high and a low densities appear locally in the recurrence plot plane, most of regions have a similar distribution density, i.e., the transference of 9-nucleotide strings in HUMHBB is well-distributed. The high and low densities display that the 9-nucleotide strings at the position close to one third of sequence length have a high frequency in the themselves positions and have a low frequency near the ending position of sequence.

To analyze local properties in the recurrence plot plane, we move to the coarse-grained recurrent plot for $k=15$. In Fig. 1(b), there exist some short and long lines, which parallel to the diagonal. The diagonal parallel lines describe that some long nucleotide strings $(>k)$ are transfered in the sequence. Especially, at the same $i$ position, several short parallel 



Fig. 1. Recurrence plots of HUMHBB for (a) 9-nucleotide strings; (b) 15-nucleotide strings.

lines with different correlation distances appear. They correspond to that a long nucleotide string is copied many times. For example, five short parallel lines near $i=5800$ correspond to that a 21 -nucleotide string $g^{2} a g^{2} c_{g a g^{2}} \mathrm{cag}^{2} a g a^{2} t c$ repeats four times, i.e., the first one exists at position 5797 to 5817 in the diagonal labeled by 1 , the second one exists at position 10783 to 10803 labeled by 2, the third one exists at position 18077 to 18097 labeled by 3, and the fourth one exists at position 66936 to 66956 la-

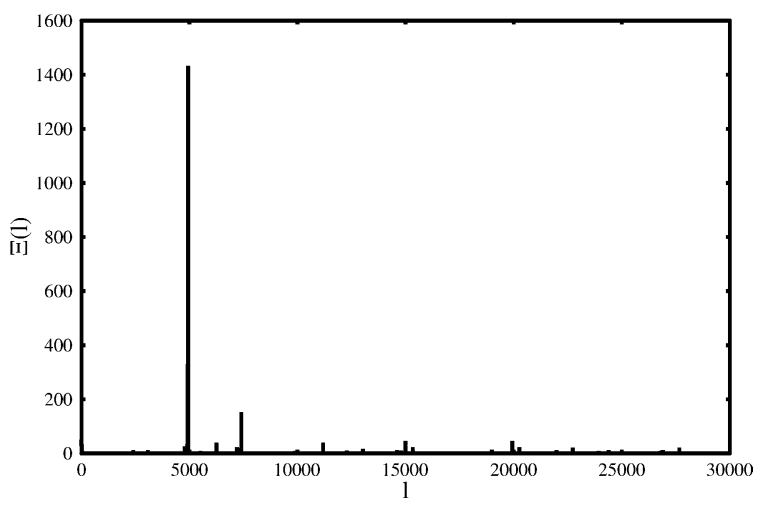

Fig. 2. A plot of correlation intensity $\Xi(l)$ versus correlation distance $l$ calculated from Fig. 1(b).

beled by 5 , as well as its subsequence, a 20-nucleotide string $g^{2} a g^{2} c t g a g^{2} \operatorname{cag}^{2} a g a^{2} t$, repeats two times, i.e., the first one occurs in the range of 5797 to 5816 in the diagonal labeled by 1 and the second one occurs in the range of 44962 to 44981 labeled by 4 . The five nucleotide strings have different correlation distance, that leads to the global transference of nucleotide strings. When many neighboring nucleotide strings are transfered with the same correlation distance, a long parallel line appears in the recurrence plot plane. It displays the local transference of nucleotide strings. From the recurrence plot, transference of long nucleotide strings can be determined. In order to preserve the total number of long nucleotide strings is not too large, we take the cut-off threshold of $30 \mathrm{nu}-$ cleotides. In Table 1 , all repeated $k(\geqslant 30)$-nucleotide strings and their correspondent positions in HUMHBB are presented. Many long nucleotide strings have correlation distances 4916 and 4936, which correspond to long parallel lines in the center of Fig. 1(b). The longest one, which has the correlation distance 4936, is a 1058-nucleotide string $t^{2} a \ldots g t g$ positioned in the ranges of 34503 to 35560 and 39439 to 40496.

Fig. 2 displays the correlation intensity $\Xi(l)$ at different correlation distance $l$ for $k=15$. When $k$ is taken as 9, the global behavior in Fig. 2 is still preserved. The maximal correlation intensity appears at $l=4936$, i.e., transference of 15 -nucleotide strings over 4936 is the most powerful. At the correlation distance $l=4916$, the correlation intensity reaches the second local maximal value. For other correlation distance, the correlation intensity is not larger than one 
Table 1

Transference of nucleotide strings with lengths $k(\geqslant 30)$ for HUMHBB

\begin{tabular}{|c|c|c|c|c|c|}
\hline No. & String & Length & Position 1 & Position 2 & $l$ \\
\hline 1 & $c t c \ldots g^{3}$ & 41 & $8032-8072$ & $44802-44842$ & 36770 \\
\hline 2 & $g^{2} t \ldots c^{3}$ & 31 & 8194-8224 & $52153-52183$ & 43959 \\
\hline 3 & $t^{2} g \ldots \operatorname{tg} a$ & 30 & 10780-10809 & $66933-66962$ & 56153 \\
\hline 4 & gtg ...atg & 30 & $13037-13066$ & $26061-26090$ & 13024 \\
\hline 5 & $a g c \ldots g c a$ & 31 & 19925-19955 & $55180-55210$ & 35255 \\
\hline 6 & $\operatorname{ctg} \ldots a g t$ & 48 & 19936-19983 & $34926-34973$ & 14990 \\
\hline 7 & $\operatorname{ctg} \ldots a g t$ & 48 & 19936-19983 & 39862-39909 & 19926 \\
\hline 8 & $a g a \ldots c^{2} a$ & 44 & $33551-33594$ & $38489-38532$ & 4938 \\
\hline 9 & $\operatorname{tg} a \ldots \operatorname{tg} a$ & 38 & 33769-33806 & $38707-38744$ & 4938 \\
\hline 10 & $t c a \ldots t^{2} a$ & 73 & $34007-34079$ & $38943-39015$ & 4936 \\
\hline 11 & $c t^{2} \ldots g^{2} t$ & 41 & $34081-34121$ & $39017-39057$ & 4936 \\
\hline 12 & $a^{3} \ldots a t c$ & 31 & $34123-34153$ & 39059-39089 & 4936 \\
\hline 13 & $a^{3} \ldots a^{3}$ & 35 & $34172-34206$ & $39108-39142$ & 4936 \\
\hline 14 & $t g^{2} \ldots g^{2} t$ & 112 & $34208-34319$ & $39144-39255$ & 4936 \\
\hline 15 & $c^{2} t \ldots g a g$ & 181 & $34321-34501$ & $39257-39437$ & 4936 \\
\hline 16 & $t^{2} a \ldots g t g$ & 1058 & $34503-35560$ & $39439-40496$ & 4936 \\
\hline 17 & $\operatorname{atg} \ldots \operatorname{tg} a$ & 43 & $34818-34860$ & $45995-46037$ & 11177 \\
\hline 18 & $c a^{2} \ldots g c t$ & 32 & $35651-35682$ & $40567-40598$ & 4916 \\
\hline 19 & $g c a \ldots g c t$ & 55 & $35717-35771$ & $40633-40687$ & 4916 \\
\hline 20 & $g^{2} t \ldots c t g$ & 175 & $35773-35947$ & $40689-40863$ & 4916 \\
\hline 21 & $a g t \ldots a g c$ & 35 & 35949-35984 & $40865-40900$ & 4916 \\
\hline 22 & $\operatorname{cag} \ldots g c t$ & 60 & $36000-36059$ & $40916-40975$ & 4916 \\
\hline 23 & $t c t \ldots t^{3}$ & 31 & $36100-36130$ & $41016-41046$ & 4916 \\
\hline 24 & $c t c \ldots g^{3}$ & 30 & $36348-36377$ & $41253-41282$ & 4905 \\
\hline 25 & $a^{3} \ldots c a^{2}$ & 31 & $37015-37045$ & $41788-41818$ & 4773 \\
\hline 26 & $\operatorname{atg} \ldots \operatorname{tg} a$ & 43 & 39754-39796 & $45995-46037$ & 6241 \\
\hline 27 & $a^{2} c \ldots a^{3}$ & 37 & $44880-44916$ & $52074-52110$ & 7194 \\
\hline 28 & $g t g \ldots g c a$ & 37 & $54761-54797$ & $62158-62194$ & 7397 \\
\hline 29 & $a g t \ldots t^{2} a$ & 40 & $54858-54897$ & $62255-62294$ & 7397 \\
\hline 30 & $a g a \ldots t c t$ & 43 & $54939-54981$ & $62336-62378$ & 7397 \\
\hline 31 & $\operatorname{ctg} \ldots t c^{2}$ & 54 & $55014-55067$ & $62413-62466$ & 7399 \\
\hline 32 & $c t c \ldots g a^{2}$ & 49 & $55069-55117$ & $62468-62516$ & 7399 \\
\hline 33 & $g^{2} t \ldots c a c$ & 47 & $55125-55171$ & $62524-62570$ & 7399 \\
\hline 34 & $c t g \ldots g t c$ & 58 & $55182-55239$ & $62581-62638$ & 7399 \\
\hline 35 & $\operatorname{tgt} \ldots \operatorname{tg} t$ & 31 & 59457-59487 & 59459-59489 & 2 \\
\hline
\end{tabular}

tenth of the maximal one. The properties give an evidence that the transference of nucleotide strings in HUMHBB has a major correlation distance.

\subsection{Correlation structure of Yeast1}

Recurrence plots of Yeast1 (Saccharomyces cerevisiae yeast, chromosome 1) with 230209 bases for $k=11$ and 15 are displayed in Fig. 3(a) and (b). Most parts of the pattern in Fig. 3(a) are similar to those in Fig. 1(a). In the comparison of coarse-grained recurrence plots, a difference of Fig. 3(b) from Fig. 1(b) is two square sets of points near the diagonal, which are labeled by 1 and 2 . A square set of points consists of many diagonal parallel lines, which correspond to many neighboring repeated nucleotide strings with a basic correlation distance. The transference of nucleotide strings is a local behavior. For example, in the square set 1 , a 95-nucleotide string $a t^{2} \ldots g^{2} t$ repeats two times at its neighboring region. They are distributed in the ranges of 25739 to 25833 and 25874 to 25968 and have a correlation distance 135 . We take the correlation distance 135 as a basic one. A 101-nucleotide string $g t a . . a c^{2}$ repeats three times at its neighboring region. The first one exists at position 25751 to 25851 , the second one exists at position 26561 

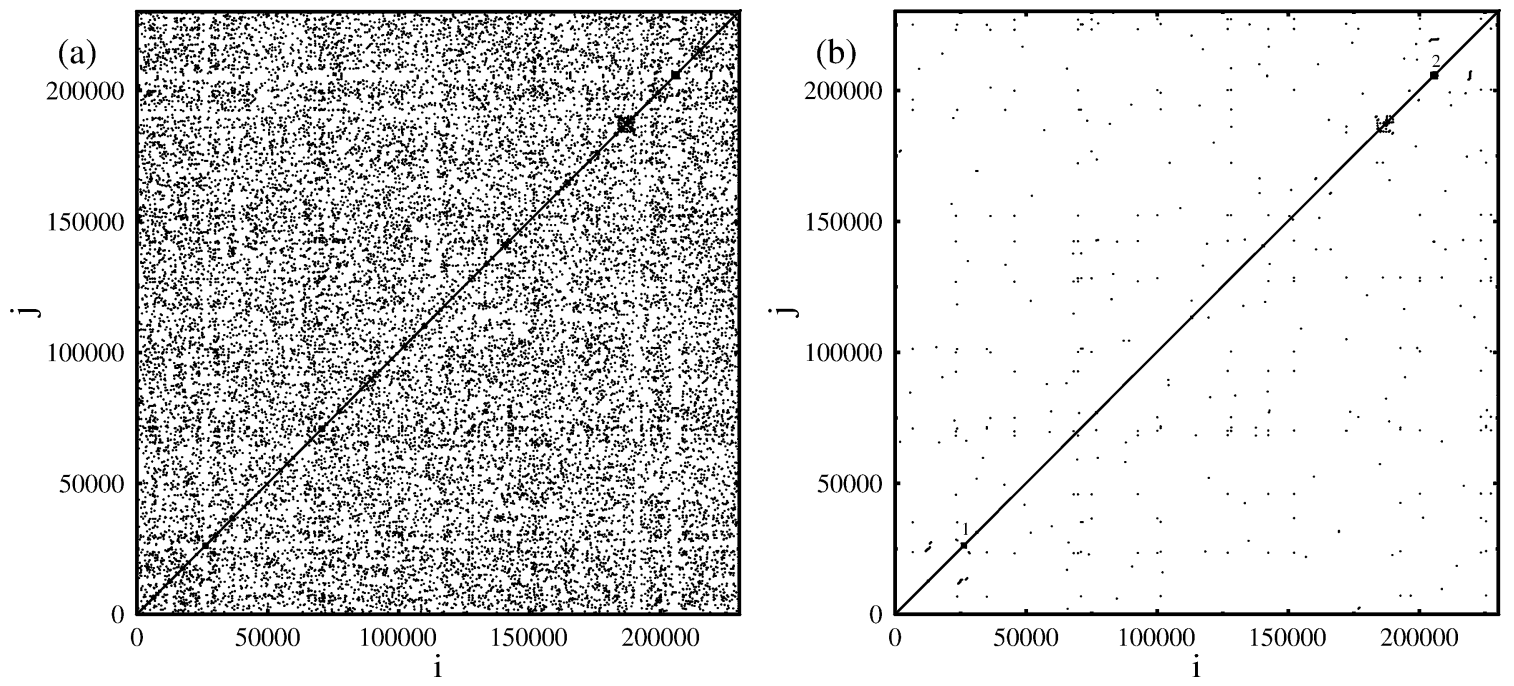

Fig. 3. Recurrence plots of Yeast1 for (a) 11-nucleotide strings; (b) 15-nucleotide strings.

Table 2

Transference of nucleotide strings with lengths $k(\geqslant 90)$ for Yeast1

\begin{tabular}{|c|c|c|c|c|c|}
\hline No. & String & Length & Position 1 & Position 2 & $l$ \\
\hline 1 & $t^{2} a \ldots a c t$ & 225 & $11745-11969$ & $24177-24401$ & 12432 \\
\hline 2 & $\operatorname{ctg} \ldots a^{2} t$ & 139 & $12258-12396$ & $24711-24849$ & 12453 \\
\hline 3 & $g^{2} a \ldots g^{2} a$ & 184 & $12988-13171$ & $25153-25336$ & 12165 \\
\hline 4 & $c^{2} g \ldots a c^{2}$ & 137 & $25715-25851$ & $26255-26391$ & 540 \\
\hline 5 & $a t^{2} \ldots g^{2} t$ & 95 & $25739-25833$ & $25874-25968$ & 135 \\
\hline 6 & $a t^{2} \ldots a c^{2}$ & 113 & $25739-25851$ & $26414-26526$ & 675 \\
\hline 7 & $g t a \ldots a c^{2}$ & 101 & $25751-25851$ & $26561-26661$ & 810 \\
\hline 8 & $g t a \ldots a c^{2}$ & 101 & $25751-25851$ & 26696-26796 & 945 \\
\hline 9 & $t^{2} g \ldots g^{2} t$ & 116 & $25853-25968$ & $26393-26508$ & 540 \\
\hline 10 & $a t^{2} \ldots g^{2} t$ & 95 & $25874-25968$ & $26279-26373$ & 405 \\
\hline 11 & $\operatorname{atg} \ldots g t g$ & 111 & $25925-26035$ & $26060-26170$ & 135 \\
\hline 12 & $\operatorname{atg} \ldots$ agt & 134 & $25925-26058$ & $26195-26328$ & 270 \\
\hline 13 & agt $\ldots g t g$ & 121 & $26050-26170$ & $26185-26305$ & 135 \\
\hline 14 & $a t^{2} \ldots g a c$ & 128 & 26279-26406 & $26414-26541$ & 135 \\
\hline 15 & $g t a \ldots g a c$ & 116 & 26291-26406 & $26561-26676$ & 270 \\
\hline 16 & $g t a \ldots g a c$ & 116 & 26291-26406 & 26696-26811 & 405 \\
\hline 17 & gta...gtg & 285 & $26426-26710$ & $26561-26845$ & 135 \\
\hline 18 & $\operatorname{tg} a \ldots a c a$ & 337 & 160239-160575 & $165827-166163$ & 5588 \\
\hline 19 & cac..tac & 285 & 204518-204802 & 204653-204937 & 135 \\
\hline 20 & $g^{2} t \ldots t a c$ & 101 & $204567-204667$ & 205512-205612 & 945 \\
\hline 21 & $g^{2} t \ldots t a c$ & 101 & 204702-204802 & 205512-205612 & 810 \\
\hline 22 & $g^{2} t \ldots a^{2} t$ & 113 & 204837-204949 & 205512-205624 & 675 \\
\hline 23 & $a c^{2} \ldots c^{2} a$ & 115 & 204855-204969 & 205395-205509 & 540 \\
\hline 24 & $c t c \ldots c a t$ & 127 & 205042-205168 & 205312-205438 & 270 \\
\hline 25 & $c a c \ldots a c t$ & 121 & 205058-205178 & 205193-205313 & 135 \\
\hline 26 & $c a c \ldots c a t$ & 111 & 205193-205303 & 205328-205438 & 135 \\
\hline 27 & $a c^{2} \ldots a^{2} t$ & 95 & 205395-205489 & 205530-205624 & 135 \\
\hline 28 & $\operatorname{atg} \ldots t^{2} c$ & 122 & 205758-205879 & 206433-206554 & 675 \\
\hline 29 & $a c^{2} \ldots t g^{2}$ & 92 & 205911-206002 & $206181-206272$ & 270 \\
\hline
\end{tabular}




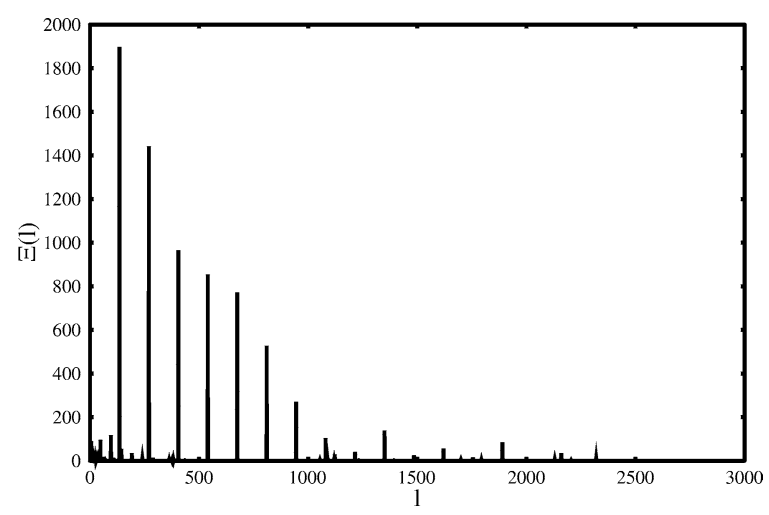

Fig. 4. A plot of correlation intensity $\Xi(l)$ versus correlation distance $l$ calculated from Fig. 3(b).

to 26661 , and the third one exists at position 26696 to 26796. Their correlation distances are 810 and 945, which are 6 and 7 times of the basic correlation distance. For the square set 2, in the same way, a 101-nucleotide string $g^{2} t \ldots t a c$ is copied three times in its neighboring region. The first one occurs in the range from 204567 to 204667 , the second one occurs in the range from 204702 to 204802, and the third one occurs in the range from 205512 to 205612. Their correlation distances are 135 and 945 , which are equal to the basic correlation distance and its 7 times. In Table 2, we present the transference of $k(\geqslant 90)$-nucleotide strings and their correspondent positions in Yeast1. In the same way, we take the cutoff threshold of 90 nucleotides. The repeated longest nucleotide string $\operatorname{tga} \ldots a c a$ has 337 letters and the correlation distance 5588. It distributes in the ranges of 160239 to 160575 and 165827 to 166163 . Many long nucleotide strings have correlation distances, which are integer times of the basic correlation distance.

Fig. 4 displays the correlation intensity $\Xi(l)$ at different correlation distance $l$ with $k=15$. The global behavior is preserved even when $k$ is changed to 11 . In a difference from Fig. 2, there exist some parallel lines with the same distance. The maximal correlation intensity appears at $l=135$. Then, when the correlation distance increases with 135 , the correlation intensity arrives at a local maximum. In the global properties, the local maximal correlation intensity decreases monotonically. The properties give an evidence that in the transference of nucleotide strings of Yeast1, its correlation distance has a constant increasing.

\section{Conclusion}

In summary, by using metric representation, recurrence plot technique is extended to analyze correlation structure of nucleotide strings in DNA sequences. In the correlation structure, some diagonal parallel lines correspond to global and local transference of nucleotide strings in the sequences. The correlation structure is quantified by correlation intensity, which can be used to display transference of nucleotide strings in the DNA sequences. It is found that, in the transference of nucleotide strings, HUMHBB has a major correlation distance, but Yeast1's correlation distance has a constant increasing.

\section{Acknowledgements}

This work was supported in part by the National Key Program for Developing Basic Science G1999032801-11. The author thanks the referee for valuable suggestions.

\section{References}

[1] H.J. Jeffrey, Nucleic Acids Res. 18 (1990) 2163.

[2] P.J. Deschavanne, A. Giron, J. Vilain, G. Fagot, B. Fertil, Mol. Biol. Evol. 16 (1999) 1391.

[3] B.-L. Hao, H.C. Lee, S.-Y. Zhang, Chaos Solitons Fractals 11 (2000) 825.

[4] J. Qi, B. Wang, B.-L. Hao, J. Mol. Evol. 58 (2004) 1.

[5] Z.-B. Wu, Electrophoresis 21 (2000) 2321.

[6] Z.-B. Wu, Fractals 11 (2003) 19.

[7] J.D. Farmer, Physica D 4 (1982) 366.

[8] J.-P. Eckmann, S.O. Kamphorst, D. Ruelle, Europhys. Lett. 4 (1987) 973.

[9] C.L. Webber, J.P. Zbilut, J. Appl. Physiol. 94 (1994) 965.

[10] P. Faure, H. Korn, Proc. Natl. Acad. Sci. USA 94 (1997) 6506.

[11] J.P. Zbilut, A. Giuliani, C.L. Webber, A. Colosimo, Protein Eng. 11 (1998) 87.

[12] H. Ochman, J.G. Lawrence, E.A. Groisman, Nature 405 (2000) 299.

[13] J.L. Bennetzen, Plant Mol. Biol. 42 (2000) 251. 